\title{
Specialized Face Perception Mechanisms Extract Both Part and Spacing Information: Evidence from Developmental Prosopagnosia
}

\author{
Galit Yovel ${ }^{1}$ and Brad Duchaine ${ }^{2}$
}

\begin{abstract}
It is well established that faces are processed by mechanisms that are not used with other objects. Two prominent hypotheses have been proposed to characterize how information is represented by these special mechanisms. The spacing bypothesis suggests that face-specific mechanisms primarily extract information about spacing among parts rather than information about the shape of the parts. In contrast, the bolistic bypothesis suggests that faces are processed as nondecomposable wholes and, therefore, claims that both parts and spacing among them are integral aspects of face representation. Here we examined these hypotheses by testing a group of developmental prosopagnosics (DPs) who suffer from deficits in face recognition. Subjects performed a face discrimination task with faces that differed either in the spacing of the parts
\end{abstract}

\section{INTRODUCTION}

Extensive evidence suggests that upright faces are processed by specialized mechanisms that are not used to process many other object classes. Studies using functional magnetic resonance imaging (fMRI) (Kanwisher, McDermott, \& Chun, 1997; McCarthy, Puce, Gore, \& Allison, 1997), event-related potentials (Eimer, 2000; McCarthy, Puce, Belger, \& Allison, 1999; Puce, Allison, \& McCarthy, 1999; Bentin, Allison, Puce, Perez, \& McCarthy, 1996), and single-cell recordings (Kreiman, Koch, \& Fried, 2001; Gross, 1992; Perrett, Rolls, \& Caan, 1982) indicate neural substrates that show face-selective responses. Neuropsychological studies have shown that face and object recognition can each be selectively impaired (Duchaine, Yovel, Butterworth, \& Nakayama, in press; Duchaine \& Nakayama, 2005; Moscovitch \& Moscovitch, 2000; Moscovitch, Winocur, \& Behrmann, 1997; Farah, 1996; Sergent \& Signoret, 1992). Finally, behavioral studies have demonstrated that faces are processed in a more configural or in a more holistic manner than objects (Farah, Wilson, Drain, \& Tanaka, 1998; Young, Hellawell, \& Hay, 1987; Tanaka \& Farah, 1993, 2003; Yin, 1969). Two hypotheses have been pro-

\footnotetext{
${ }^{1}$ Tel-Aviv University, ${ }^{2}$ University College, London
}

but not the parts (spacing task), or in the parts but not the spacing of the parts (part task). Consistent with the holistic hypothesis, DPs showed lower performance than controls on both the spacing and the part tasks, as long as salient contrast differences between the parts were minimized. Furthermore, by presenting similar spacing and part tasks with houses, we tested whether face-processing mechanisms are specific to faces, or whether they are used to process spacing information from any stimulus. DPs' normal performance on the tasks of two houses indicates that their deficit does not result from impairment in a general-purpose spacing mechanism. In summary, our data clearly support face-specific bolistic hypothesis by showing that face perception mechanisms extract both part and spacing information.

posed to characterize the nature of specialized face representation: the face-specific spacing bypothesis and the face-specific holistic hypothesis.

\section{Face-specific Spacing Hypothesis}

Studies have shown that we are highly sensitive to subtle displacements of face parts (e.g., eyes, nose, and mouth) in upright faces (Haig, 1984) but not in faces that are inverted or in faces with negative contrast (Kemp, McManus, \& Pigott, 1990). Such findings led several researchers to suggest that face perception mechanisms are special in that they extract a precise distance among face parts (i.e., spacing), whereas information about the characteristics of parts is represented by general object recognition mechanisms (for a review, see Maurer, Grand, \& Mondloch, 2002).

This face-specific spacing hypothesis initially drew support from studies examining face inversion effect. The face inversion effect refers to a much larger effect that orientation inversion has on face recognition compared to object recognition. Studies that have employed the face inversion effect to test the spacing hypothesis have used two sets of face stimuli (Mondloch, Le Grand, \& Maurer, 2002; Le Grand, Mondloch, Maurer, \& Brent, 2001; Freire, Lee, \& Symons, 2000). For the spacing 
sets, the face parts (e.g., eyes and mouth) were identical in all faces, but the spacing of the parts varied. Conversely, in the part sets, the face parts varied, but the spacing of those parts remained constant. These faces were presented in a same-different matching task in an upright and in an inverted orientation. If face perception mechanisms are specialized for the processing of spacing but not for the processing of part-based information, inversion should result in a larger cost for matching spacing information rather than for matching part-based information.

Several studies found support for this prediction. In the first published study, Freire et al. (2000) found a very large inversion effect for discrimination of spacing information (26\%) but found no inversion effect for discrimination of part-based information. Other studies have also found greater inversion effects for spacing information than for part information (Mondloch et al., 2002; Le Grand et al., 2001). These findings suggest that distinct mechanisms mediate the processing of information about spacing among parts and the identity of parts. Furthermore, because faces are represented in a qualitatively different manner than are objects, it has been assumed that the mechanisms extracting spacing information are specialized for faces, whereas those used for part representation are used with a wide range of objects.

This account predicts that individuals with impaired face recognition may show deficits in processing spacing but not deficits in part-based information from faces. Support for this prediction was found in a group of subjects who suffered from infantile cataracts that were removed in their first year of life. Although low-level visual abilities in these subjects were intact, they showed face recognition difficulties in adulthood. When tested with a face discrimination task involving part and spacing information, these individuals performed part discriminations normally, but were impaired with spacing discriminations (Le Grand, Mondloch, Maurer, \& Brent, 2003; Le Grand et al., 2001). Similar results were recently found in adults with Williams syndrome (Karmiloff-Smith et al., 2004). The dissociability of these processes gained more support when it was shown that children develop sensitivity to spacing changes more slowly than they develop sensitivity to part changes (Mondloch et al., 2002). Note that these forgoing studies used the same face exemplar known as the Jane face (Le Grand et al., 2001). However, despite apparently clear results supporting the face-specific spacing hypothesis, some recent studies have challenged it.

\section{Face-specific Holistic Hypothesis}

The face-specific holistic hypothesis suggests that faces are processed as nondecomposed wholes and that face parts are processed interactively rather than independently. Thus, in contrast to the spacing bypothesis, the bolistic bypothesis predicts that information about face parts and spacing among parts is processed by a common mechanism. Consistent with the holistic hypothesis, three studies with normal subjects have found that inversion has comparable effects on spacing and part discrimination in faces (Malcolm, Leung, \& Barton, 2005; Riesenhuber, Jarudi, Gilad, \& Sinha, 2004; Yovel \& Kanwisher, 2004a). These findings are consistent with earlier studies that examined the recognition of face parts (i.e., eyes, nose, and mouth) in the context of a face or in isolation (whole-part effect) (Tanaka \& Sengco, 1997; Tanaka \& Farah, 1993). These studies found that when subjects discriminated face parts in the context of a whole face, performance was lower when faces were presented in an inverted orientation than when they were presented in an upright orientation. Thus, in contrast to the spacing hypothesis, these findings suggest that parts in upright faces are processed by a mechanism different from that used for parts in inverted faces.

Several other studies provide support for the holistic hypothesis and suggest that face mechanisms extract both spacing-based and part-based information. In an fMRI study, Yovel and Kanwisher (2004a) investigated whether face-selective brain areas respond differently to part and spacing information in faces; they found no difference in responses to spacing and parts. Additionally, similar to the behavioral findings, the fMRI inversion effect (i.e., lower response to inverted than upright faces) was similar for the spacing and part task. Furthermore, Yovel and Kanwisher (2004b) examined individual differences in performance for part and spacing discrimination and found that normal subjects showed a large positive correlation between performance on spacing and part discrimination tasks for upright faces but not for inverted faces or houses. These findings suggest that a common mechanism is used to process information about spacing and parts for faces, whereas distinct mechanisms are used for the discrimination of spacing and parts for nonfaces. All of these findings suggest that special mechanisms used with faces represent information about both parts and spacing among parts and are, therefore, inconsistent with the face-specific spacing hypothesis.

\section{Domain-general Spacing Hypothesis}

The two hypotheses discussed above both suggest that face perception, not object perception, is carried out by different mechanisms. Such face-specific hypotheses are motivated by demonstrations that faces and other types of objects are dissociable (Duchaine et al., in press; Moscovitch et al., 1997). However, in any experiment showing a dissociation between faces and objects, recognition of faces can only be compared to a limited number of object classes. It may be that the object classes used in such experiments did not demand spacing processing like faces did. As a result, this leaves open the possibility that the mechanisms that process faces are not facespecific but are domain-general mechanisms that operate 
on any class with diagnostic spacing information. According to this general spacing hypothesis, impairments with face spacing discriminations will be accompanied by nonface spacing discriminations (Behrmann, Avidan, Marotta, \& Kimchi, 2005; Levine \& Calvanio, 1989).

One direct way to examine the domain-general spacing bypothesis is to compare the magnitude of the inversion effect for spacing and part discriminations with faces to other object classes. Such a comparison is essential because an inversion effect by itself is not informative with respect to the nature of specialized face-processing mechanisms. Only an inversion effect that is larger for faces than for nonfaces can provide information about the possibility of specialized face-processing mechanisms. Yovel and Kanwisher (2004a) manipulated spacing-based and part-based information in house stimuli in the same way they manipulated faces. They found large inversion effects for face parts and face spacing, but they found no inversion effects for house part or house spacing discriminations. Furthermore, the fMRI response of fusiform face area (FFA) showed a similar response for part and spacing discrimination in houses, and the response was much lower than the response to faces. These findings suggest that the spacing processing applied to faces is not used with other classes of objects.

\section{Developmental Prosopagnosia as a Test Case for the Three Hypotheses}

The conflicting results discussed above make it difficult to determine which hypothesis best characterizes the mechanisms performing face recognition. In this study, our goal was to directly address these three hypotheses by testing individuals who show severe face perception deficits. These individuals are expected to show difficulties with all tasks that are normally mediated by intact face-processing mechanisms. Thus, to test the predictions of each hypothesis, we tested developmental prosopagnosics (DPs) on perceptual matching tasks with faces and houses that differed in part or in spacing information.

DPs are individuals who failed to develop normal face recognition abilities (Behrmann \& Avidan, 2005). There are a variety of causes of this condition, including genetic deficits (Behrmann et al., 2005; Duchaine \& Nakayama, 2005; Duchaine, 2000; Bentin, Deouell, \& Soroker, 1999; de Haan, 1999), early brain damage (Barton, Cherkasova, Press, Intriligator, \& O'Connor, 2003), and early visual problems (Le Grand, Mondloch, Maurer, \& Brent, 2004; Le Grand et al., 2001, 2003). Difficulties with other visual abilities, such as recognition of facial expressions and objects, sometimes accompany the face recognition impairments of DPs, but some cases appear to be quite selective (Duchaine et al., in press; Duchaine \& Nakayama, 2005; Duchaine, Dingle, Butterworth, \& Nakayama, 2004; Nunn, Postma, \& Pearson, 2001; Bentin et al., 1999).

To resolve the conflicting results from face discrimination tasks, we used two sets of face stimuli (see Figure 1). One set, referred to here as Alfred, yielded similar inversion effects in controls for the spacing and part tasks (Yovel \& Kanwisher, 2004a); these results
Figure 1. The face and house stimuli were varied in two ways. For the part set, the eyes and mouth in the face and the windows and door in the house were replaced by similar parts from different exemplars. The spacing of the parts was not changed. Conversely, to create the spacing set, the position of the eyes and mouth or the position of the door and windows was varied. These manipulations were determined in a pilot study based on changes that generated average performance level in the dynamic range (about $75 \%$ ) across the spacing and part conditions of faces and houses.

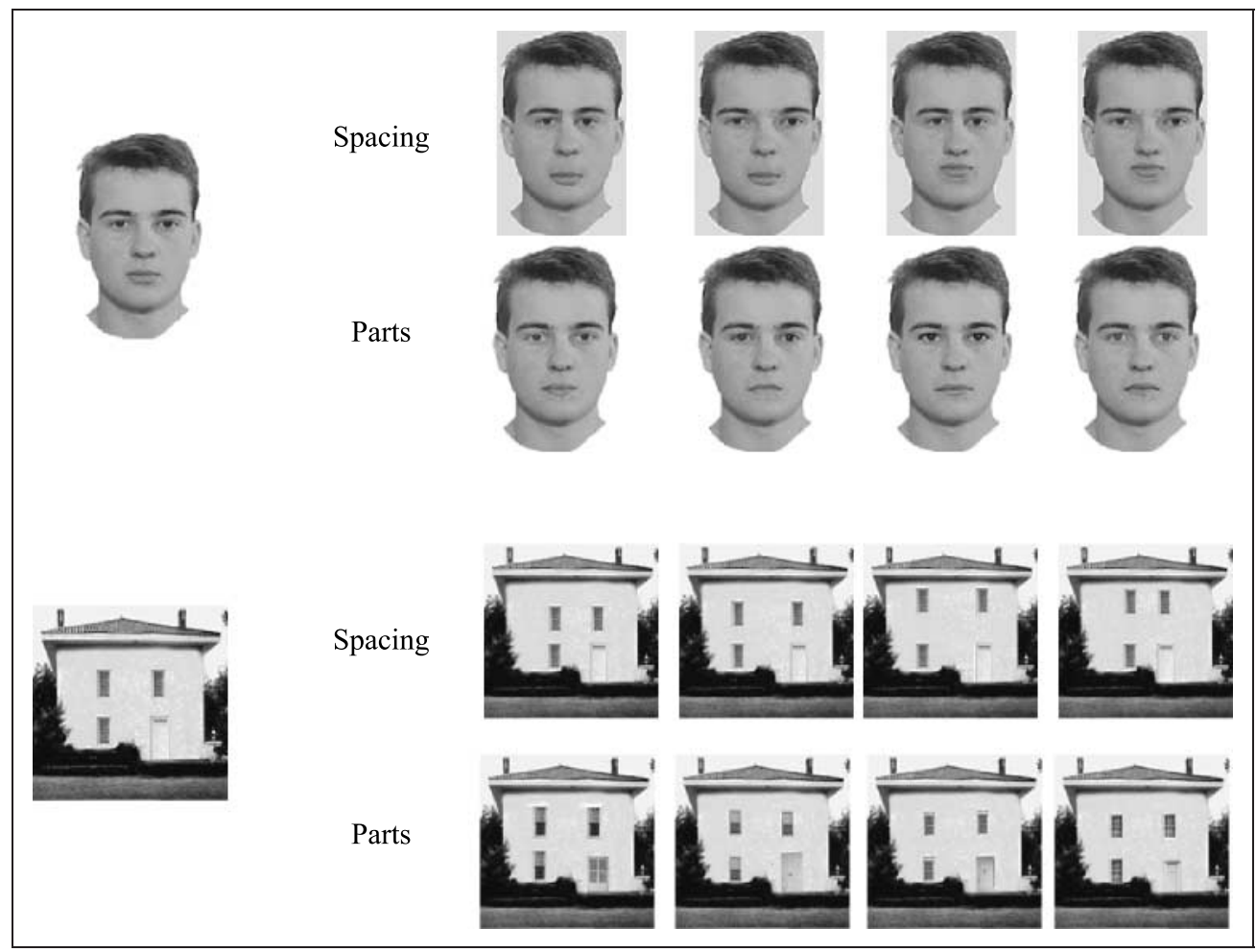


support the face-specific holistic hypothesis. The other set, known as the Jane face, was used in experiments that revealed a larger inversion effect for the spacing task than the part task in normal controls (Mondloch et al., 2002; Le Grand et al., 2001). The Jane faces have been also used in studies with patients with infantile cataract (Le Grand et al., 2001, 2003, 2004), in a study of adults with Williams syndrome (Karmiloff-Smith et al., 2004), and in a developmental study that found dissociation between spacing and parts (Mondloch et al., 2002). In addition, we tested the prosopagnosics with a house discrimination task with identical task demands to address the domain of spacing processes used with faces.

The predictions of the different hypotheses are presented in Figure 2. In (A), the face-specific spacing hypothesis predicts that prosopagnosics will be impaired with face spacing items, but they will be normal with other discriminations. In (B), the face-specific holistic hypothesis predicts that prosopagnosics will be

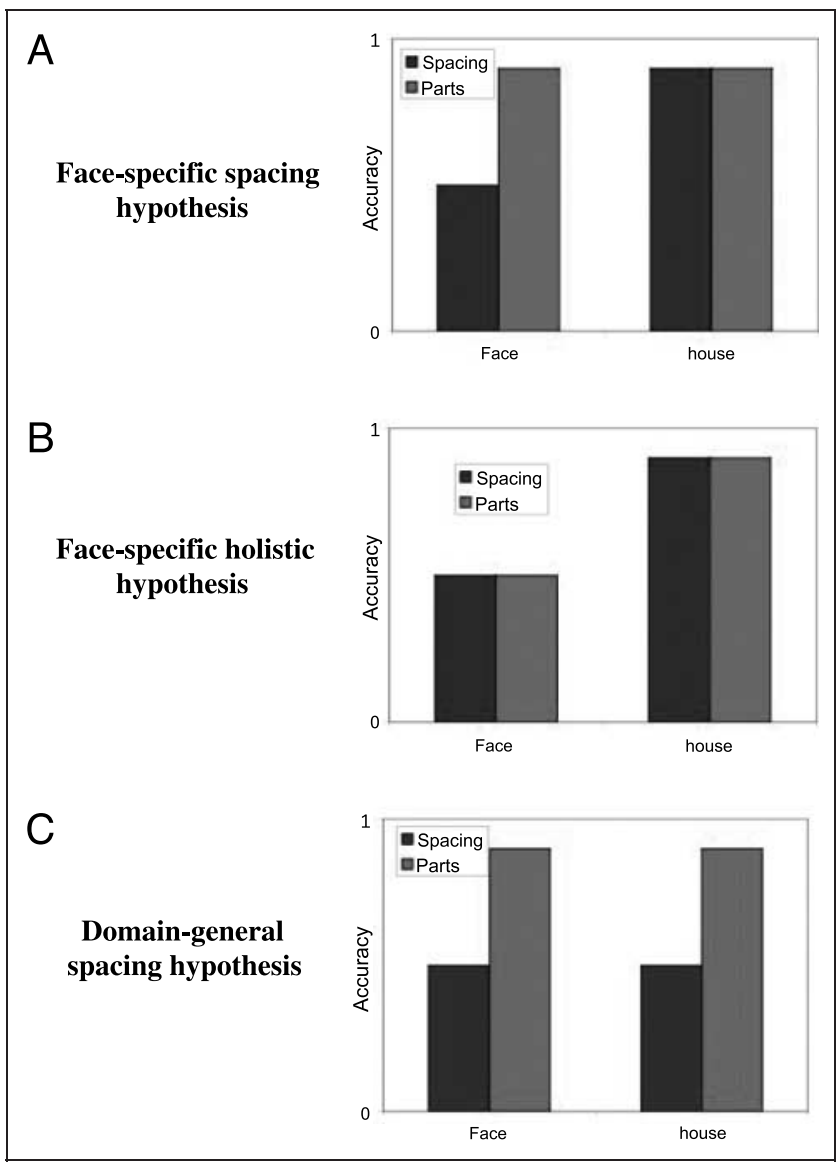

Figure 2. Three hypotheses were tested. (A) The face-specific spacing hypothesis predicts that DPs will show a deficit with spacing information only for faces. (B) The face-specific holistic hypothesis predicts that DPs will show a deficit on both the part and spacing tasks for faces, but intact performance for houses. (C) The domain-general spacing hypothesis predicts that DPs will show deficits in processing spacing information from both faces and houses. impaired with both face part and face spacing, but they will perform normally with houses. In (C), the domaingeneral spacing hypothesis predicts that prosopagnosics will be impaired with spacing discriminations with both faces and houses, but they will perform normally for both types of part discriminations.

\section{RESULTS}

\section{Experiment 1}

Eight subjects with developmental prosopagnosia and 34 controls participated in Experiment 1 . Table 1 displays their standardized scores on three face recognition

Table 1. Standardized Scores of Each of the DPs on the Three Face Recognition Tasks

\begin{tabular}{cccccc}
\hline & & & Face & Face & \\
Age & Famous & Old- & Old- & \\
DPs & Sex (years) & Faces 1 & New A & New RT & CFMT \\
\hline
\end{tabular}

Experiment 1: Alfred-Jane experiments

$\begin{array}{lcccccc}\text { A.C. } & \text { M } & 20 & -4.8 & -10.6 & -1.7 & -2.5 \\ \text { D.D. } & \text { F } & 32 & -4.7 & -4.3 & -8.4 & -2.3 \\ \text { Edward } & \text { M } & 53 & -10.1 & -6.4 & -4.2 & -2.4 \\ \text { J.H. } & \text { M } & 23 & -12.5 & -7.2 & -5.1 & -2.6 \\ \text { K.L. } & \text { F } & 46 & -6.5 & -4.1 & -5 & -2.9 \\ \text { L.A. } & \text { F } & 40 & -4.9 & -3 & -6.2 & -2 \\ \text { M.L. } & \text { M } & 24 & -15.3 & -6.4 & -4 & -2.4 \\ \text { R.S. } & \text { F } & 27 & -4 & -5.6 & 0 & -2.6 \\ \text { Control mean } & & 23.6 / 22.6 & 0.96 & 971 \mathrm{msec} & 80.4 \% \\ \text { Control } & \text { SD } & & 1.4 / 1.9 & 0.03 & 205 & 11\end{array}$

Experiment 2: Ann experiments

\begin{tabular}{lccrrrl} 
A.M. & F & 64 & -0.2 & -2.5 & -9.0 & -1.9 \\
K.M. & F & 21 & -2.5 & -2.0 & -2.7 & -1.9 \\
D.D. & F & 32 & -5.1 & -4.3 & -8.4 & -2.3 \\
N.Z. & F & 21 & -5.7 & -3.5 & -4.2 & -3.8 \\
J.W. & M & 25 & -7.5 & -9.1 & -6.2 & -3.7 \\
B.K. & M & 56 & -3.6 & -4.7 & -7.5 & -1.9 \\
Control mean & & 52.5 & 0.96 & $971 \mathrm{msec}$ & $80.4 \%$ \\
Control SD & & 6.6 & 0.03 & 205 & 11 \\
\hline
\end{tabular}

$Z$ scores were computed by subtracting the control average from each DP's percentage of correct responses and then dividing by the control standard deviation [(DP value - control average)/control $S D$ ]. Values worse than the control mean are negative. For Famous Face 1, two averages and two standard deviations are presented. The first is for college-age controls, whereas the second is for adults $45-55$ years of age. A.C. was not tested with the famous face test used, but scored 4.8 standard deviations below the mean on Famous Faces 2 .

$\mathrm{RT}=$ reaction time; $\mathrm{CFMT}=$ Cambridge Memory Face Test 
tasks. The tests and the control subjects are described in Methods. The DPs had accuracy scores that were two standard deviations or more below the control mean on all three tests (see Table 1).

\section{Accuracy}

We first describe results with Alfred faces, which yield similar inversion effects for the spacing and part tasks in controls (Yovel \& Kanwisher, 2004a). This face was constructed such that performance levels are away from ceiling or floor and are similar for upright spacing and part discriminations (see Methods for further information). Figure 3 shows proportions that are correct for part and spacing discriminations with Alfred faces and house stimuli. If we first consider the control results (presented on the right side of the figure), we see that performance was matched across all conditions so that the difficulty of each discrimination was comparable. In addition, because the mean proportion of correct responses ranged from $78 \%(S D=10.0 \%)$ to $79 \%(S D=$ $9.6 \%$ ), ceiling or floor effects had little or no influence on control scores.

The results for the DPs show a pattern very different from that of the controls, and this pattern is consistent with the predictions of the face-specific holistic hypothesis (see Figure 2B). The DPs' average performance was similar to that of the controls on the house task, but it was much worse than that of the controls on the face task: Group $\times$ Stimulus interaction, $F(1,39)=20.37, p<$ .0001 . Whereas controls averaged $78.5 \%$ on the two face discriminations, DPs averaged 57.4\%. In contrast, the DPs showed good performance with the houses (75\%)

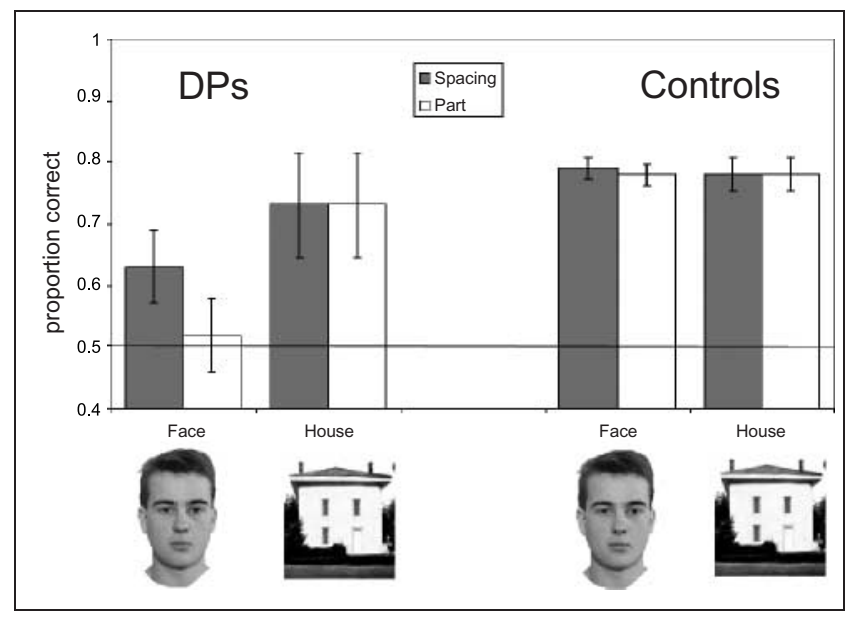

Figure 3. Proportion of correct responses for the DPs and the controls on the face and house tasks. For the controls, the proportion of correct responses was similar for all conditions. Consistent with the face-specific holistic hypothesis, DPs show deficits on both part and spacing discriminations of faces, but normal performance on the house tasks. Error bars represent the standard error of the difference between the part and spacing conditions for faces and houses. The solid line indicates chance performance level. relative to the controls (78\%). Notably, the DPs, like the controls, showed no differences in performance on the spacing and part tasks for either faces or houses: the three-way Group $\times$ Stimulus $\times$ Task interaction was not significant, $F(1,40)=1.82, p=.19$. Thus, their performance with spacing and part discriminations is inconsistent with predictions of the face-specific spacing hypothesis and the domain-general spacing hypothesis.

Because there is often substantial variability among DPs, we created Figure 4 to display individual DP results. It displays performance level for each DP and the average performance of the control group (larger diamond) for the part and spacing tasks for faces (A) and for houses (B). The individual results with faces make it clear that individual DPs tended to show a similar pattern, as the average results in that performance with both spacing and part discriminations were usually poor. In contrast, nearly all of the DPs were within the normal range with both types of discriminations with houses. These findings suggest that impairment in developmental prosopagnosia is neither a general deficit in processing spacing information in faces or nonfaces (Figure 2C), nor is it a face-specific deficit in processing spacing information (Figure 2A) but is a specific face-processing impairment affecting both spacing-based and part-based information (Figure 2B).

\section{Reaction Times}

Although the primary dependent measure in our study was accuracy, we also reported reaction time (RT) data. Analysis of RT for correct responses (trimmed $200 \mathrm{msec}<$ RT $<1500 \mathrm{msec})$ showed slower RTs to the faces for DPs (846 msec) than for controls $(754 \mathrm{msec})$, but the difference did not reach significance, $F(1,40)=3.22$, $p=$ .08. RTs were slower for the face part (801 msec) than the face spacing (741 msec) tasks for both controls and DPs: main effect of Task, $F(1,40)=18.84, p<.0001$. More important, Group $\times$ Task interaction was not significant, $F(1,40)<1$, which suggests that DPs showed a pattern of response to the part and spacing face tasks similar to that of controls.

There was no difference between DPs and controls in RTs on the house tasks. RTs were faster on the house part tasks ( $765 \mathrm{msec}$ ) than on the house spacing tasks (729 msec); main effect of Task, $F(1,39)=5.01, p<.05$, for both DPs and controls. The Group $\times$ Task interaction was not significant, $F(1,39)<1$, which suggests that DPs and controls showed a pattern of response to the part and spacing house tasks similar to that of controls.

\section{Resolving Discrepancies with Previous Studies: Comparing Alfred and Jane}

Our data show comparable impairments for the DPs with part and spacing processing with the Alfred faces. 


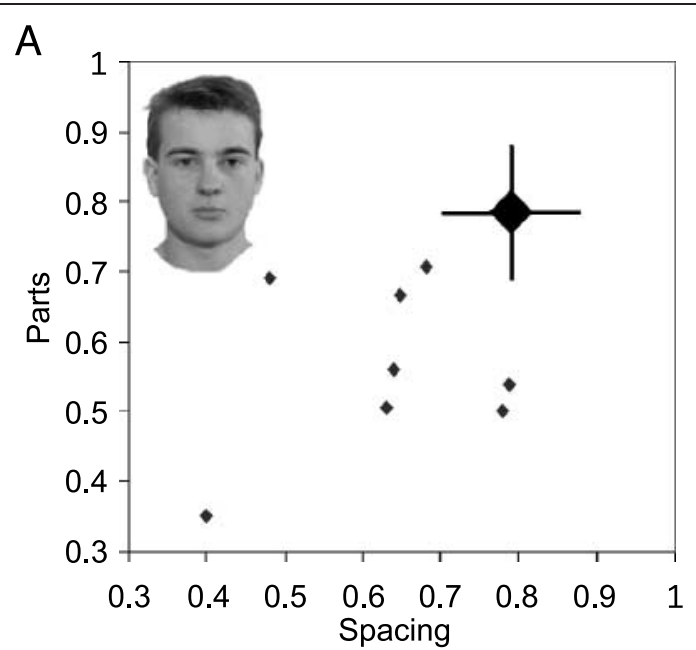

B

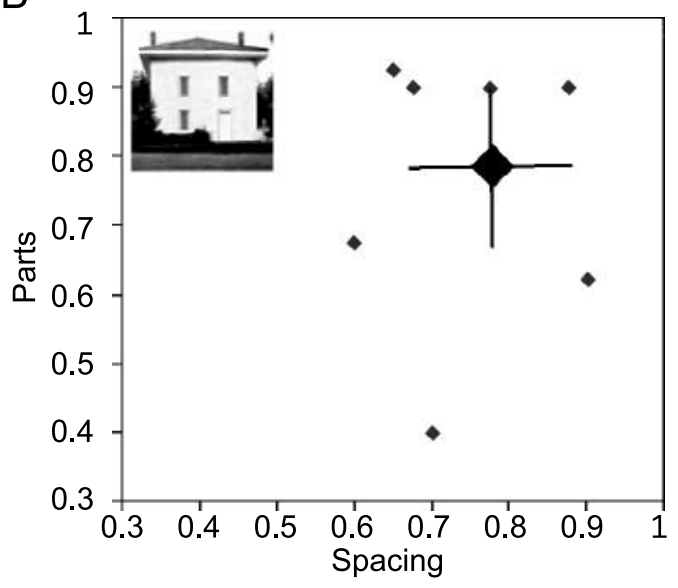

Figure 4. The performance of each DP is presented along with the average performance of controls (large diamond) on the (A) face tasks and on the (B) house tasks. Error bars represent one standard deviation above and below the mean for the spacing and part tasks in the control group (see Appendix I).

In contrast, studies that used the Jane faces found that individuals with face-processing deficits have impairments with spacing but not with part-based face information (Karmiloff-Smith et al., 2004; Le Grand et al., 2001). There are a number of differences between these studies that may account for these inconsistent findings.

First, this discrepancy could simply be the result of testing groups with qualitatively different face recognition impairments. For instance, it could be that the DPs that we have tested have impairments with both dissociable mechanisms hypothesized to process face spacing and face parts, whereas individuals with face difficulties due to visual deprivation are only impaired with the mechanism used with face spacing. Another possible explanation for the discrepancy is that the spacing and part discriminations with Alfred were pre- sented in a mixed design, whereas the part and spacing tasks with Jane faces were blocked. Other task parameters, such as exposure duration, may also underlie these differences.

Thus, to assess whether differences between groups or task parameters are responsible for the discrepancy, we compared how the DPs in our study performed with Alfred and Jane discriminations in a mixed design. The DPs should perform similarly with Alfred and Jane if the discrepancy is caused by one of the above-mentioned possibilities. However, if our DPs show better performance on the part task than on the spacing task with the Jane faces, it will indicate that the discrepancy is due to specific characteristics of the face stimuli that have been used in the conflicting experiments.

\section{Accuracy}

In our face experiment, we included the same Jane faces that were used in past experiments (Karmiloff-Smith et al., 2004; Mondloch et al., 2002; Le Grand et al., 2001, 2003). This allows us to directly compare the Alfred faces with the Jane faces under the same experimental conditions in the same sample of subjects. Figure 5 displays the proportion of correct responses for the spacing and part tasks with Alfred and Jane faces for the DPs and the controls. As is evident from the figure, the DPs' performance showed a different pattern with Jane and Alfred faces. Our controls, like those in other studies with Jane faces (Karmiloff-Smith et al., 2004; Mondloch et al., 2002; Le Grand et al., 2001, erratum), scored better on the part discriminations (88\%) than on the spacing discriminations (74\%). Like Le Grand et al. (2001), performance for the Jane part task did not differ between the DPs (83\%) and the controls (88\%), $t(40)=2.72, p=.11$. Again, as with the patients with cataract and subjects with Williams syndrome, the DPs performed more poorly with the Jane spacing task (62\%) than controls (74\%), $t(40)=$ 8.67, $p<.006$. This dissociation between discrimination of parts and spacing is inconsistent with our findings with Alfred faces, which yielded lower performance for DPs than for controls on both the part tasks, $t(40)=30.78, p<.0001$, and the spacing tasks, $t(40)=14.42, p<.001$. Accordingly, an analysis of variance (ANOVA) on proportions of correct responses with Group as a between-subject factor and with Task and Face Stimuli (Alfred, Jane) as a repeated measure factor yielded a significant three-way Group $\times$ Face Stimulus $\times$ Task interaction, $F(1,40)=4.73, p<.05$ (see Figure 5).

Figure 6 shows the performance of each DP and the average performance of the control group on the spacing and part tasks for the Jane and Alfred faces. Whereas the DPs are shifted down relative to the control mean on both the spacing axis and the part axis with Alfred faces, we see that they are shifted down only on the 
Figure 5. The proportion of correct responses of DPs and controls on the discrimination of spacing and parts in Alfred and Jane. Consistent with previous reports that used the Jane faces, we find higher performance on the part tasks than on the spacing tasks for both controls and DPs. However, when performance on the part and spacing tasks is matched away from ceiling effects in controls (Alfred faces), DPs show deficits on both face tasks. Error bars represent the standard error of the difference between the part and spacing conditions for faces and houses. The solid line indicates chance performance level.

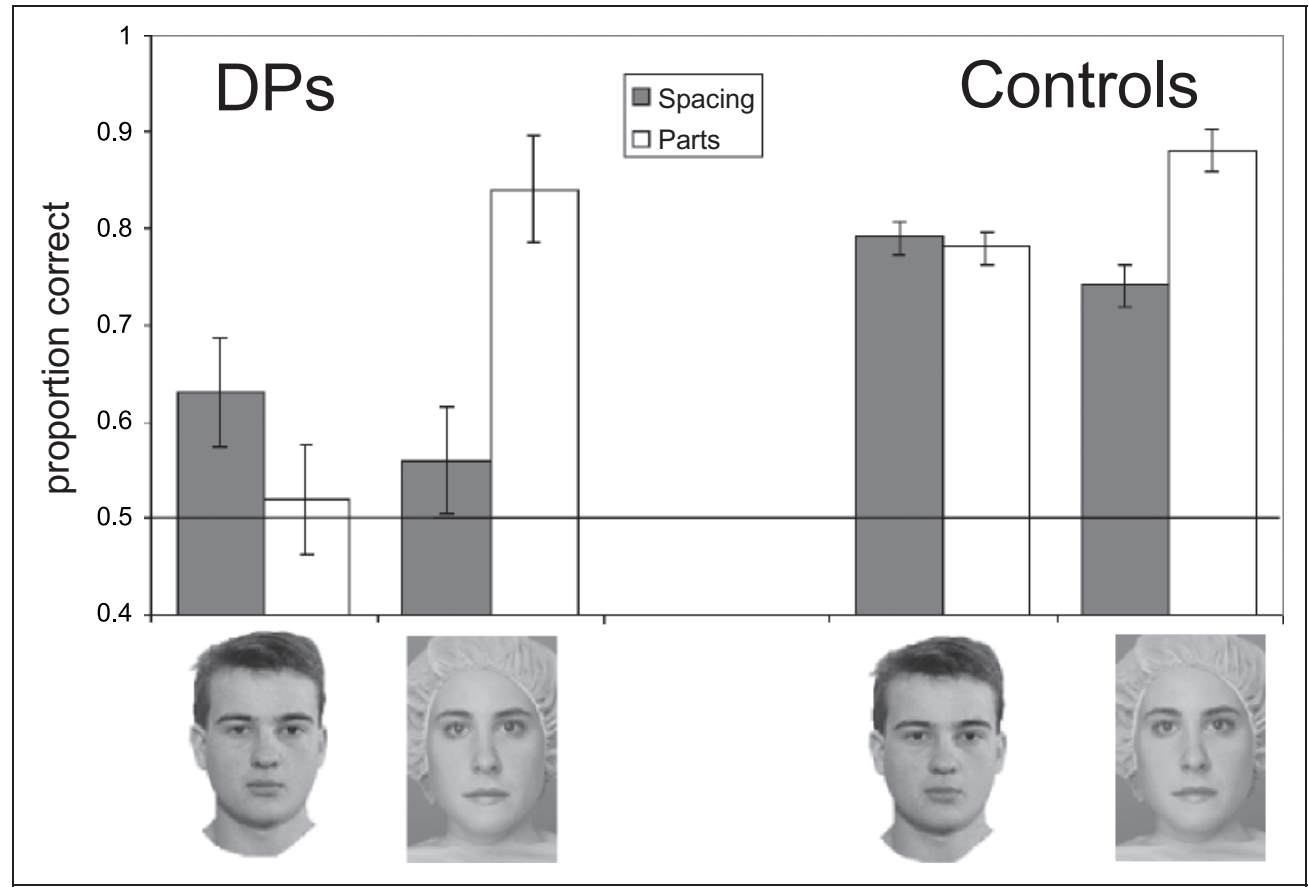

spacing axis with Jane faces. On the Jane task, the control group and seven of eight DPs showed a higher percentage of correct responses on the part task than on the spacing task. Thus, our data for the Jane faces are consistent with previous reports that used these faces. More important, these data show that the discrepancies between our findings with Alfred faces and prior findings with Jane faces are not due to differences between our subject groups or specific task parameters, but lie in the nature of the face stimulus sets that were used in the different studies. We will consider these differences and their implications in Experiment 2.

\section{Reaction Times}

Consistent with the accuracy results, response times were faster for the Jane part task $(731 \mathrm{msec})$ than for the Jane spacing task (805 msec), $F(1,40)=6.43, p<$ .02 , for both controls and DPs. Whereas DPs were worse than controls on the spacing tasks but not on the part tasks for accuracy data, DPs were slower than controls on both the part tasks (848 $\mathrm{msec}$ ) and the spacing tasks (898 msec): main effect of Task, $F(1,40)=10.84$, $p<.005$, but no Group $\times$ Task interaction. Because Le Grand et al. (2001) did not report the response time data of their prosopagnosic group, we cannot determine whether our findings with Jane are in agreement with their observations.

In summary, our findings show that prosopagnosic individuals showed deficits in both spacing-based and part-based processing for Alfred faces but not for Jane faces. In Experiment 2, we investigate the source of the discrepancy between the two face exemplars.

\section{Experiment 2}

One important difference between the manipulation of part information in Alfred and Jane faces was that Alfred part changes primarily involved the shape of the face parts. In contrast, the Jane part changes involved the shape of the parts and salient changes in the brightness and contrast of the different parts. The most notable brightness/contrast difference is that two of the women are wearing eyeliner or lipstick, whereas two are not. Leder and Bruce (2000) have shown that discrimination of faces that differ in color information does not produce the face inversion effect, which suggests that such information does not involve specialized face-processing mechanisms.

To resolve the discrepancies between the results we obtained with the Jane face and with the Alfred face, we generated a new face (Ann), which, like Jane, was a female face in which the hair was cropped. The Ann face was manipulated in three ways (see Figure 7 and Methods):

1. Spacing: Similar to the spacing manipulation of the Alfred and Jane faces, we generated four faces that differed in the distance between the eyes and the distance between the nose and the mouth.

2. Part-SC (shape + contrast) ("Jane-like"): The eyes and mouth from four different faces replaced the eyes and mouth of the original face. Like the Jane part manipulation, the parts differed in both shape and contrast/brightness (e.g., lipstick). 

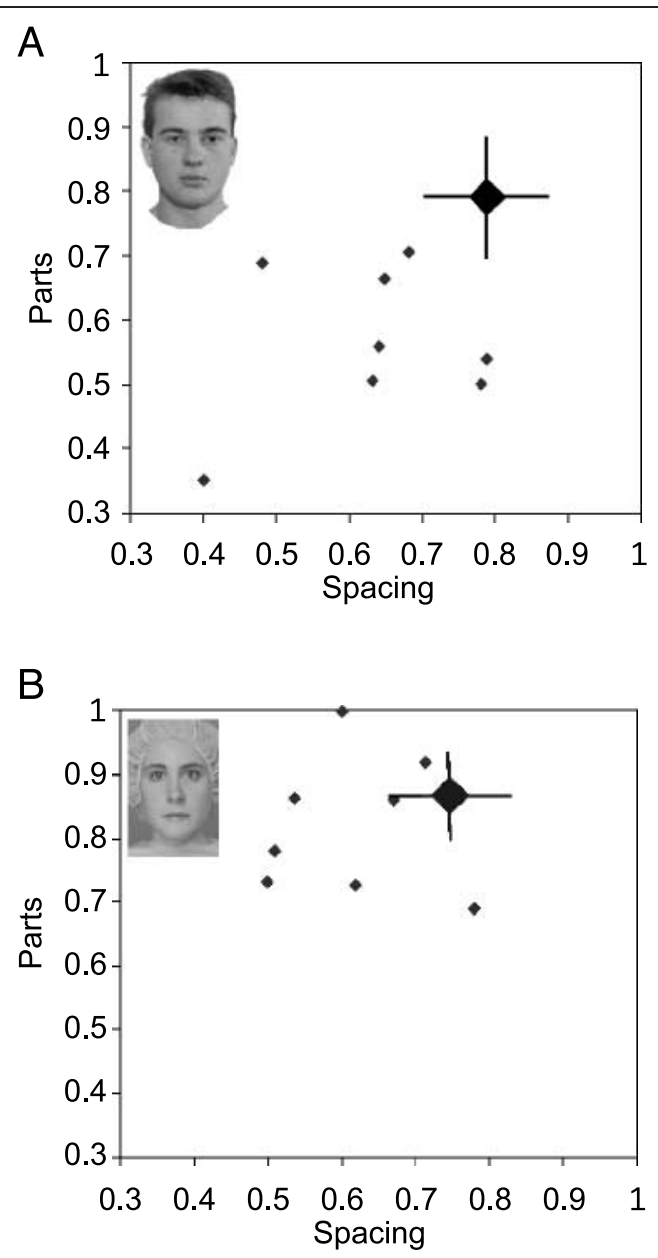

Figure 6. Performance on the (A) Alfred task and on the (B) Jane task for each DP is presented along with the average performance of controls (large diamond). Error bars represent one standard deviation above and below the mean for each task in the control group (see Appendix I).

3. Part-S (shape) ("Alfred-like"): The eyes and mouth from four different faces replaced the eyes and mouth of the original face, but differences in contrast/ brightness were minimal.

We hypothesized that face mechanisms are not necessary to discriminate faces that differ in salient contrast/ brightness information, which can be performed by lower-level visual processing mechanisms. Therefore, we expect no inversion effect in controls and no deficit in discrimination of the part-SC Ann task in prosopagnosic subjects. In contrast, we hypothesized that face mechanisms are as necessary for the discrimination of parts that primarily differ in shape as they are for the extraction of spacing information. Thus, we expect normal subjects to show an inversion effect in the part-S Ann task that is as large as in the spacing task, and we predict that prosopagnosic subjects will have difficulty in discriminating faces that differ in such part information.
Six subjects with developmental prosopagnosia and 15 controls participated in Experiment 2. Five of these subjects did not participate in Experiment 1. Table 1 displays their standardized scores on three face recognition tasks.

\section{Face Inversion Effect in Controls}

Subjects first performed a discrimination task with the spacing and part-S tasks, followed by a discrimination task that included the spacing and part-SC tasks. Control subjects were presented with both upright and inverted face tasks. The prosopagnosic subjects performed only the upright face tasks.

Consistent with our predictions, we found an inversion effect in the spacing and part-S (Alfred-like) tasks, but not in the part-SC (Jane-like) task (Figure 8A). The Type (spacing, part-S, part-SC) by Orientation (upright, inverted) interaction was highly significant, $F(2,14)=13.23, p<.0001$, which reflects a significantly larger inversion effect for part-S than for the part-SC task, $F(2,14)=14.75, p<.001$, and for spacing than for the part-SC task, $F(2,14)=20.15, p<.001$, but no difference in the magnitude of the inversion effect for the part-S and spacing tasks, $F(2,14)<1$ (Figure 8A).

\section{Performance of Prosopagnosic Subjects}

Consistent with the inversion effect findings, prosopagnosic subjects performed significantly worse in the spacing task (prosopagnosics, 64\%; controls, 78\%), $t(1,19)=3.76, p<.001$, and in the part-S task (prosopagnosics, 68\%; controls, 80\%), $t(1,19)=2.98, p<.01$, than the controls. However, they did not differ from the control group in their performance for the part-SC task (prosopagnosics, 84\%; controls, 85\%) (Jane-like), $t(1,19)=0.11 p>.90$ (Figure 8B). These findings suggest that prosopagnosics can discriminate faces that differ in brightness/contrast as well as controls, but not between faces that primarily differ in the shape of the part.

\section{DISCUSSION}

It is often argued that specialized face perception mechanisms primarily process the spacing among face parts, but not the parts themselves (Maurer et al., 2002; Leder \& Bruce, 2000). The data we present in this article challenge this idea. We found that individuals who suffer from developmental prosopagnosia show face discrimination deficits for faces that differ in spacing among parts and for faces that differ in parts in which contrast/brightness differences are minimal. This inversion effect demonstrates that the stimulus has en- 
Figure 7. The three Ann sets used in Experiment 2. The Ann spacing set was used in both blocks involving Ann faces. Eye spacing and the distance between the nose and mouth were manipulated. In the Alfred-like part-S set, the eyes and mouth were varied by substituting eyes and mouths that differed in shape, but not in contrast/brightness. In contrast, the eyes and mouths in the Jane-like part-SC set varied in shape and contrast/ brightness. By inverting the figure, readers can experience the effect of inversion on the different sets.

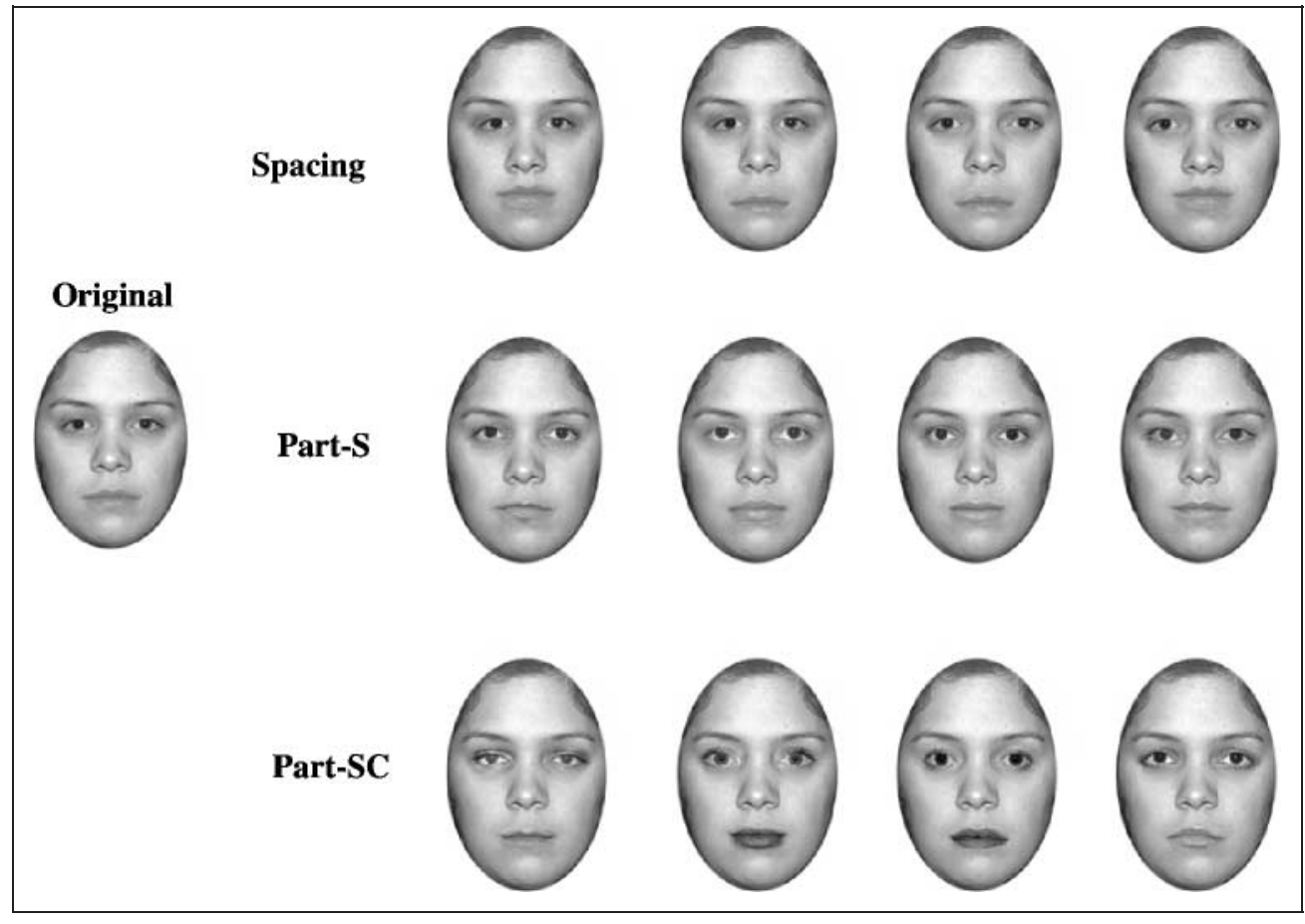

gaged face-specific mechanisms. Thus, these findings suggest that specialized face perception mechanisms process both part and spacing information. Our findings are consistent with recent reports of a similar-sized inversion effect on part and spacing discrimination tasks for faces (Malcolm et al., 2005; Riesenhuber et al., 2004; Yovel \& Kanwisher, 2004a) and a similar fMRI response in face-selective brain areas (i.e., FFA) for the spacing and part tasks (Yovel \& Kanwisher, 2004a).

In addition, our results indicate that the deficit that these DPs show on the discrimination of part and spacing information is not a general deficit in the extraction of spacing information because they performed as well as controls did in a closely matched task with houses (Figure 3). This dissociation is consistent with Yovel and Kanwisher (2004a), who found an inversion effect for the spacing and part tasks for faces but not for houses, and a similar FFA response on both tasks for houses that was lower than that for faces. Thus, our data are inconsistent with the general spacing bypothesis.

Our findings of a similar impairment for both spacing and part processing in DPs are inconsistent with reports that used the Jane faces to study part and spacing discrimination in neuropsychological subjects (Karmiloff-Smith et al., 2004; Le Grand et al., 2001). These individuals showed a deficit in processing spacing-based but not part-based face information. One important difference between the manipulation of part information in Alfred and Jane was that Alfred part changes involved primarily the shape of the face parts. In contrast, the Jane part changes involved sa- lient changes in brightness/contrast. The most notable brightness/contrast differences are that two of the women are wearing eyeliner, whereas two are not, and two are wearing lipstick, whereas two are not. To directly address the inconsistent findings with the two face sets, we generated a new face stimulus that was designed to be like the Jane-part (part-SC) set and the Alfred-part (part-S) set (see Figure 7). In other words, in the Janelike manipulation, the faces differed in both shape and contrast/brightness information, whereas in the Alfredlike part manipulation, contrast differences between the face parts were minimized and the parts primarily differed by shape. Our findings clearly show that control subjects did not show inversion effect on this task. These findings are consistent with those of Leder and Bruce (2000), who reported no inversion effect for the discrimination of faces that differed in color information. Furthermore, we found that our prosopagnosic subjects did as well as controls in discriminating these faces. In contrast, when contrast/brightness differences across the faces were minimized, the controls showed a similar inversion effect on the spacing and part tasks, and prosopagnosics were as impaired on the part task as on the spacing task. Thus, intact face perception mechanisms are not necessary for discriminating faces that differ by salient contrast information. However, they are as necessary for the representation of shapebased part information as they are for the representation of spacing information. This accounts for the observation that prosopagnosics often report that they are able to recognize people by way of distinctive facial characteristics, such as Mikhail Gorbachev's port-wine stain. 
Figure 8. Results for Experiment 2 involving the Ann faces. (A) The inversion effect for normal subjects in the three conditions. Error bars represent the standard error of the difference between the upright and inverted conditions. (B) A comparison of the performance of the normal subjects and the prosopagnosics for the three conditions. Error bars represent the standard error of the difference between the DPs and the control group.

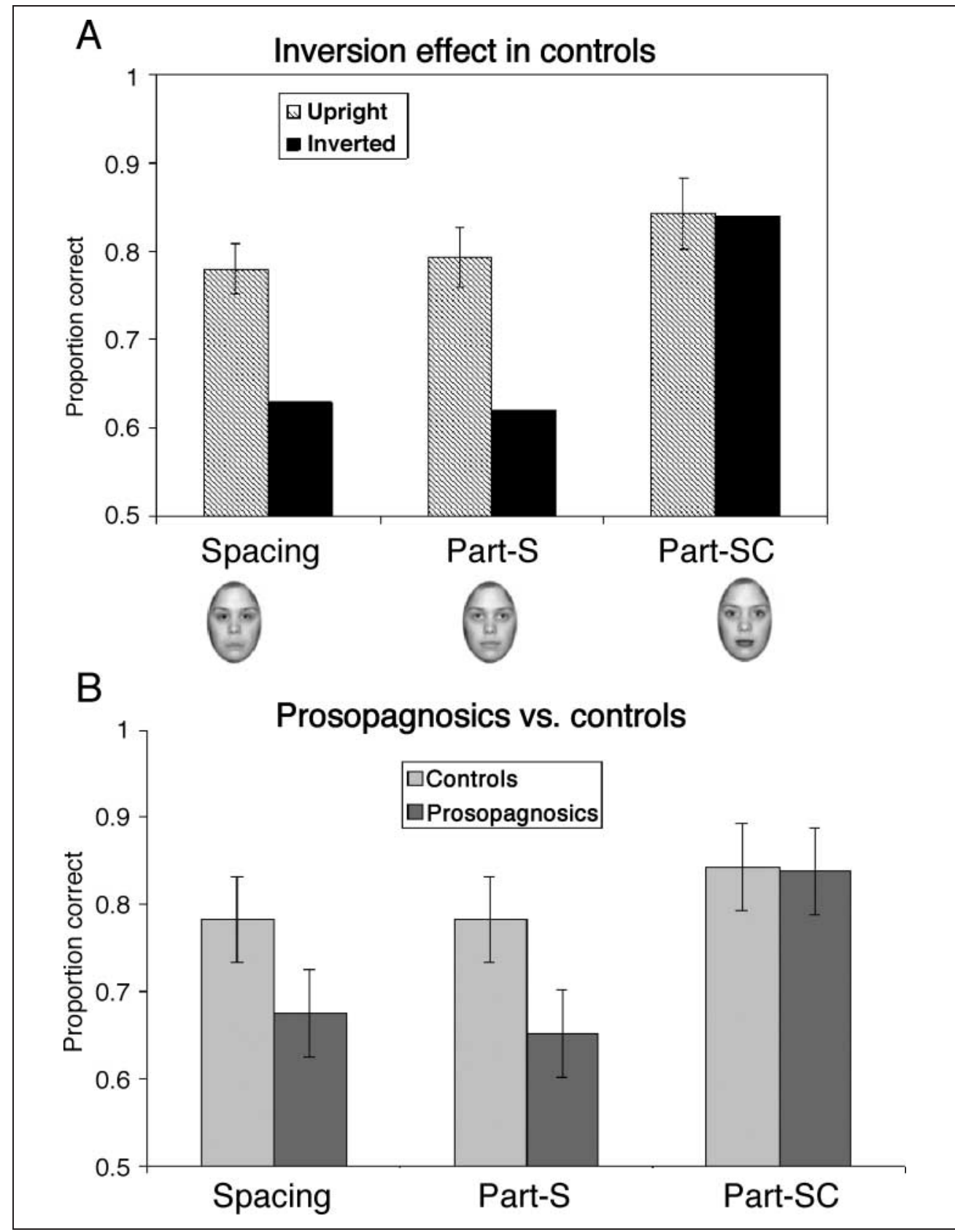

In conclusion, our results suggest that specialized face perception mechanisms extract both part and spacing information from faces only. These findings challenge the spacing hypothesis but support holistic theories of face perception, which suggest that face perception mechanisms are special in that they interactively process facial information as a nondecomposable whole.

\section{METHODS}

\section{Prosopagnosic Subjects}

We tested 13 prosopagnosics individuals who contacted the Center for Prosopagnosia Research at the Harvard
University Web site (www.faceblind.org) because they reported significant problems with face recognition in daily life. None except R.S. was aware of any events that may have caused brain damage, and all reported lifelong problems with face recognition. R.S. reported that she collided with a refrigerator as a young child, and although she remained conscious, her mother told her that immediately after the collision, R.S. asked, "Why are the trees white?" as she looked out the window. Four of the DPs have been reported on in other articles, and these articles and their designation in these articles are listed in Appendix II. Eight prosopagnosics participated in Experiment 1. One prosopagnosic from this group (D.D.) and five new prosopagnosics participated in 
Experiment 2. Each subject was tested with a battery of face-processing tasks in order to evaluate their faceprocessing abilities. To determine whether they did, in fact, suffer from face recognition impairments, we tested them with three types of tests.

\section{Famous Faces}

Two different famous face tests were used with the two groups. Subjects in Experiment 1 were presented with photos of 25 celebrities drawn from entertainment and politics (Duchaine et al., in press; Duchaine, 2000), whereas those in Experiment 2 were presented with 60 faces. The images were cropped so that little hair or clothing was visible, and each image was presented for $10 \mathrm{sec}$. Two groups of controls were used for the test with 25 images. College-age controls were compared to DPs in their twenties, and controls between 45 and 55 years of age were compared to older DPs. The college-age controls averaged $23.6(S D=1.4)$ on the test, and the middle-aged adult controls averaged 22.6 ( $S D=1.9)$. Controls for the 60 -face test were between 35 and 45 years of age, and they averaged 52.5 $(S D=6.6)$.

\section{Cambridge Memory Test for Faces}

In the test, subjects were introduced to six target faces with study images (Duchaine \& Nakayama, 2006). Each face is shown in three different views; immediately after presentation of those study images, subjects were presented with three forced-choice items, each of which consisted of one of the study images paired with two other faces in the same pose. After this introductory phase, subjects were tested with 54 forced-choice items consisting of novel views of one of the target faces along with two nontarget faces. Fifty college-age subjects served as our controls, and they averaged $80.4 \%$ $(S D=11.0)$. We also tested nine adults with a mean age of 47 years, and their average was slightly higher than that of the college students; thus, to be conservative, all DPs were compared to the college-age group.

\section{Face Old-New Recognition Memory Tests}

In each old-new recognition memory test, 40 faces were used (Duchaine \& Nakayama, 2005). Ten faces were target faces, and they were shown twice during the study phase of the experiment. During the test phase, each target face was shown twice along with 30 nontarget faces. Two versions of the test, which used separate sets of faces, were created. Grayscale yearbook photographs of women's faces were cropped so that very little or no hair was visible. Graduate students in their twenties to mid-thirties served as controls. Although these controls are not age-matched with Edward, K.L., A.M., and B.K., all scored normally on a number of parallel object recognition tests when compared to the same control group (for Edward's data, see Duchaine et al., in press; for K.L.'s data, see Duchaine \& Nakayama, 2005). A' was used as the measure of discrimination, and the control mean was $0.96(S D=.03)$. An average $\mathrm{A}^{\prime}$ score and RT were computed for each subject (except J.W.) by combining performance on the two versions of the test. J.W. was only tested with one of the tests, but we were unable to retest him. His scores on all face tests were so poor that it is clear that he is prosopagnosic.

Standardized scores for each prosopagnosic are presented in Table 1. The face recognition impairments of prosopagnosics are evident from their extremely poor scores.

\section{Control Subjects for Experiments 1 and 2}

The control group in Experiment $1(N=34)$ that is included in this report is a subset of the control group that was reported in Yovel and Kanwisher (2004a). Stimulus order in Yovel and Kanwisher was counterbalanced, and the controls used in this article were those who, like the DPs, performed the face task before the house task. Fifteen age-matched control subjects (age, 18-54 years) participated in Experiment 2.

\section{Stimuli and Apparatus}

Photoshop was used to create the spacing and part sets for the face and house stimuli. Stimuli were presented using Superlab 1.2. Subjects viewed the stimuli from a distance of approximately $50 \mathrm{~cm}$. The stimuli subtended $4.6^{\circ}$ of visual angle in width and $7.4^{\circ}$ in length.

\section{Face Stimuli}

Alfred face stimuli. Two sets of four face stimuli were generated from a picture of a male face. For the spacing set, four faces, in which eyes were either closer to or farther apart from each other and in which the mouth was either closer to or farther from the nose, were constructed. For the part set, the two eyes and the mouth were replaced in each of the four faces by eyes and mouths of a similar shape from different original face photos. By piloting the part and spacing manipulations prior to the current experiment, we determined manipulations that produced a similar average performance for the part and spacing tasks and that were in the dynamic range away from ceiling or floor effects. Figure 1 shows a face stimulus generated by the same procedure, which yielded behavioral findings similar to those of the face stimulus used in the experiment (which is not presented in the figure because we did not obtain permission to publish it). 
Jane face stimuli. The Jane face stimuli were adapted from the studies conducted by the Maurer laboratory (Mondloch et al., 2002; Le Grand et al., 2001; Freire et al., 2000). In contrast to the Alfred stimuli, the part and spacing tasks with the Jane stimuli were not matched for performance, and performance on the part task was significantly higher than performance on the spacing task.

Ann face stimuli. Three sets of faces (spacing, part-S, and part-SC), which each included four different stimuli, were generated. For the spacing set, four faces, in which the eyes were either closer to or farther apart from each other and in which the mouth was either closer to or farther from the nose, were constructed. For the part-SC set, the eyes and mouth were replaced in each of the four faces by eyes and mouths that differed in both shape and contrast/brightness information (makeup). For the part-S set, the eyes and mouth were replaced in each of four faces by eyes and mouths that differed in shape, but contrast differences were minimized. By piloting the part and spacing manipulations prior to the current experiment, we determined manipulations that produced a similar average performance for the part-S task and the spacing task that were in the dynamic range away from ceiling or floor effects.

House stimuli. House stimuli were created using a method similar to that used for the face stimuli. For the spacing set, four houses were constructed in which we manipulated the location of the windows and the door (the two left-side windows and the right-side window and door were closer together or farther apart, or the two upper windows were closer to or farther from the roof). For the part set, the windows and the door were replaced by windows and a door of similar overall shape but of a different texture (see Figure 1). Pilot studies determined manipulations that produced similar performance levels on the part and spacing tasks that were comparable to those of the face task.

\section{Procedure}

Experiment 1

Subjects were presented with a sequential same-different matching task. Each trial started with a 500-msec fixation dot at the center of the screen. A first stimulus was presented for $250 \mathrm{msec}$ followed by a 1000-msec interstimulus interval during which time the fixation dot was on the screen. The second face was on the screen for $250 \mathrm{msec}$. The part and spacing trials were presented in a randomized order within each block. The face task preceded the house task for both the DPs and the controls. Trials with Alfred and Jane were randomly interleaved. Subjects were not informed that the stimuli would differ in spacing or parts. Each task (upright face, upright house) included a total of 80 stimuli: 20 pairs of different stimuli and 20 pairs of the same stimuli for the part and the spacing tasks.

\section{Experiment 2}

The procedure for the Ann task was similar to the procedure used for the Alfred task except for the following differences: To obtain a performance level of 75\% correct in the Ann task, the face stimuli were presented for $500 \mathrm{msec}$. Pilot studies showed that when the part-SC trials were mixed in the same block with the part-S trials, normal subjects' performance with part-S and spacing tasks was too low to allow valid tests of the inversion effect (floor effects). However, when part-SC trials were presented in a separate block, the proportion of correct responses for the part-S task and the spacing task improved and allowed us to validly examine the effect of inversion. It seems that the salient difference between the faces in the partSC manipulation (see Figure 7) modified subjects' discrimination criterion, and we missed the more subtle differences between the spacing and the part-S manipulations relative to the case in which these stimuli were presented in a separate block. Thus, we presented the two part tasks in separate blocks. In each block, the part trials were randomly mixed with the spacing trials. Subjects were not informed about the nature in which the face stimuli were manipulated. Note that all controls and prosopagnosic subjects were tested with the same procedure, so the blocking (or lack thereof in Experiment 1) cannot account for the conditions in which the prosopagnosics showed impaired performance.

\section{Data Analysis}

\section{Experiment 1}

We computed the proportion of correct responses and RT for the spacing and part tasks for faces and houses. ANOVA was performed with Group (DP, controls) as a between-subject factor and with Stimulus (face, house) and Task (part, spacing) as within-subject factors.

\section{Experiment 2}

We computed the proportion of correct responses for the spacing task, the Jane-like part (part-SC), and the Alfred-like part (part-S) tasks. A repeated measures ANOVA with Orientation and Type (spacing, part-SC, and part-S) as within-subject factors was performed to compare the inversion effects for the three stimulus manipulations in controls. 


\section{APPENDIX I}

Performance of each of the DPs on the face and house tasks, average performance, and standard deviation of a control group $(n=34)$.

\begin{tabular}{|c|c|c|c|c|c|c|}
\hline & \multicolumn{2}{|c|}{ Houses } & \multicolumn{2}{|c|}{ Alfred Face } & \multicolumn{2}{|c|}{ Jane Face } \\
\hline & Spacing & Parts & Spacing & Parts & Spacing & Parts \\
\hline A.C. & 0.70 & 0.40 & 0.40 & 0.35 & 0.60 & 1.00 \\
\hline Edward & 0.68 & 0.90 & 0.48 & 0.69 & 0.51 & 0.78 \\
\hline K.L. & 0.65 & 0.93 & 0.68 & 0.71 & 0.54 & 0.86 \\
\hline L.A. & 0.78 & 0.90 & 0.79 & 0.54 & 0.71 & 0.92 \\
\hline M.L. & 0.60 & 0.68 & 0.63 & 0.51 & 0.50 & 0.73 \\
\hline R.S. & 0.88 & 0.90 & 0.64 & 0.56 & 0.78 & 0.69 \\
\hline D.D. & 0.90 & 0.63 & 0.78 & 0.50 & 0.67 & 0.86 \\
\hline J.H. & - & - & 0.65 & 0.67 & 0.62 & 0.73 \\
\hline Average & 0.74 & 0.76 & 0.63 & 0.55 & 0.62 & 0.83 \\
\hline \multicolumn{7}{|l|}{ Controls } \\
\hline Average & 0.78 & 0.78 & 0.79 & 0.78 & 0.74 & 0.88 \\
\hline$S D$ & 0.12 & 0.11 & 0.10 & 0.10 & 0.10 & 0.08 \\
\hline
\end{tabular}

\section{APPENDIX II}

Four of the DPs have been reported on in other articles. Next to each subject's designation in this article, we list other articles that they have appeared in, along with their designation in those articles (in parentheses).

J.H.: Duchaine \& Nakayama, 2005, Journal of Cognitive Neuroscience (M1)

Edward: Duchaine et al., in press, Cognitive Neuropsychology (Edward); Duchaine et al., 2004, Neuron (Edward)

K.L.: Duchaine \& Nakayama, 2005, Journal of Cognitive Neuroscience (F2)

M.L.: Duchaine \& Nakayama, 2005, Journal of Cognitive Neuroscience (M2)

\section{Acknowledgments}

We thank Daphne Maurer, Cathy Mondloch, and Richard Le Grand for providing us with the Jane face stimuli. We are thankful to Alison Harris and Javid Sadr for their suggestions. Ken Nakayama and Nancy Kanwisher provided invaluable support for this project. We also thank Arielle Tambini, Kathleen Cui, Nao Gamo, Gayle Speck, and Kerry Dingle for their help with control data collection, and Stephanie Chow and Kathleen Cui for help with stimulus generation. This work was supported by grants from the National Institutes of Health (F32 MH64246-02 and RO1 EY13602).

Reprint requests should be sent to Galit Yovel, Department of Brain and Cognitive Sciences, Massachusetts Institute of Technology, 77 Massachusetts Avenue, NE20-443, Cambridge, MA 02139, or via e-mail: galit@mit.edu.

\section{REFERENCES}

Barton, J. J., Cherkasova, M. V., Press, D. Z., Intriligator, J. M., \& O'Connor, M. (2003). Developmental prosopagnosia: A study of three patients. Brain and Cognition, 51, 12-30.

Behrmann, M., \& Avidan, G. (2005). Congenital prosopagnosia: Face-blind from birth. Trends in Cognitive Sciences, 9, 180-187.

Behrmann, M., Avidan, G., Marotta, J. J., \& Kimchi, R. (2005). Detailed exploration of face-related processing in congenital prosopagnosia: 1. Behavioral findings. Journal of Cognitive Neuroscience, 17, 1130-1149.

Bentin, S., Allison, T., Puce, A., Perez, E., \& McCarthy, G. (1996). Electrophysiological studies of face perception in humans. Journal of Cognitive Neuroscience, 8, 551-565.

Bentin, S., Deouell, L. Y., \& Soroker, N. (1999). Selective visual streaming in face recognition: Evidence from developmental prosopagnosia. NeuroReport: An International Journal for the Rapid Communication of Research in Neuroscience, 10, 823-827.

de Haan, E. (1999). A familial factor in the development of face recognition deficits. Journal of Clinical and Experimental Neuropsychology, 21, 312-315.

Duchaine, B., \& Nakayama, K. (2005). Dissociations of face and object recognition in developmental prosopagnosia. Journal of Cognitive Neuroscience, 17, 249-261.

Duchaine, B., \& Nakayama, K. (2006). The Cambridge Face Memory Test: Results for neurologically intact individuals and an investigation of its validity using inverted face stimuli and prosopagnosic subjects. Neuropsychologia, 44, 576-585.

Duchaine, B., Yovel, G., Butterworth, E. J., \& Nakayama, K. (in press). Prosopagnosia as an impairment to face-specific recognition mechanisms: Elimination of the testable domain-general hypotheses. Cognitive Neuropsychology.

Duchaine, B. C. (2000). Developmental prosopagnosia with normal configural processing. NeuroReport, 11, 79-83.

Duchaine, B. C., Dingle, K., Butterworth, E., \& Nakayama, K. (2004). Normal greeble learning in a severe case of developmental prosopagnosia. Neuron, 43.

Eimer, M. (2000). Event-related brain potentials distinguish processing stages involved in face perception and recognition. Clinical Neurophysiology, 111, 694-705.

Farah, M. J. (1996). Is face recognition "special"? Evidence from neuropsychology. Behavioural Brain Research, 76, 181-189.

Farah, M. J., Wilson, K. D., Drain, M., \& Tanaka, J. N. (1998). What is "special" about face perception? Psychological Review, 105, 482-498.

Freire, A., Lee, K., \& Symons, L. A. (2000). The face-inversion effect as a deficit in the encoding of configural information: Direct evidence. Perception, 29, 159-170.

Gross, C. G. (1992). Representation of visual stimuli in inferior temporal cortex. Philosophical Transactions of the Royal Society of London, Series B, Biological Sciences, 335, 3-10.

Haig, N. D. (1984). The effect of feature displacement on face recognition. Perception, 13, 505-512.

Kanwisher, N., McDermott, J., \& Chun, M. M. (1997). The fusiform face area: A module in human extrastriate cortex specialized for face perception. Journal of Neuroscience, 17, 4302-4311.

Karmiloff-Smith, A., Thomas, M., Annaz, D., Humphreys, K., Ewing, S., Brace, N., Duuren, M., Pike, G., Grice, S., \& Campbell, R. (2004). Exploring the Williams syndrome face-processing debate: The importance of building developmental trajectories. Journal of Child Psychology and Psychiatry, 45, 1258-1274. 
Kemp, R., McManus, C., \& Pigott, T. (1990). Sensitivity to the displacement of facial features in negative and inverted images. Perception, 19, 531-543.

Kreiman, G., Koch, C., \& Fried, I. (2001). Category-specific visual responses of single neurons in the human medial temporal lobe. Nature Neuroscience, 3, 946-953.

Le Grand, R., Mondloch, C. J., Maurer, D., \& Brent, H. P. (2001). Neuroperception. Early visual experience and face processing. Nature, 410, 890.

Le Grand, R., Mondloch, C. J., Maurer, D., \& Brent, H. P. (2003). Expert face processing requires visual input to the right hemisphere during infancy. Nature Neuroscience, 6, 1108-1112.

Le Grand, R., Mondloch, C. J., Maurer, D., \& Brent, H. P. (2004). Impairment in holistic face processing following early visual deprivation. Psychological Science, 15, $762-768$.

Leder, H., \& Bruce, V. (2000). When inverted faces are recognized: The role of configural information in face recognition. Quarterly Journal of Experimental Psychology, A, Human Experimental Psychology, 53, 513-536.

Levine, D. N., \& Calvanio, R. (1989). Prosopagnosia: A defect in visual configural processing. Brain and Cognition, 10, 149-170.

Malcolm, G., Leung, C., \& Barton, J. J. S. (2005). Regional variations in the inversion effect for faces: Differential effects for feature shape, spatial relations, and external contour. Perception, 33, 1221-1231.

Maurer, D., Grand, R. L., \& Mondloch, C. J. (2002). The many faces of configural processing. Trends in Cognitive Sciences, 6, 255-260.

McCarthy, G., Puce, A., Belger, A., \& Allison, T. (1999). Electrophysiological studies of human face perception: II. Response properties of face-specific potentials generated in occipitotemporal cortex. Cerebral Cortex, 9, 431-444.

McCarthy, G., Puce, A., Gore, J. C., \& Allison, T. (1997). Face-specific processing in the human fusiform gyrus. Journal of Cognitive Neuroscience, 9, 605-610.

Mondloch, C. J., Le Grand, R., \& Maurer, D. (2002). Configural face processing develops more slowly than featural face processing. Perception, 31, 553-566.

Moscovitch, M., \& Moscovitch, D. A. (2000). Super face-inversion effects for isolated internal or external features, and for fractured faces. Cognitive Neuropsychology, 17, 201-219.

Moscovitch, M., Winocur, G., \& Behrmann, M. (1997). What is special about face recognition? Nineteen experiments on a person with visual object agnosia and dyslexia but normal face recognition. Journal of Cognitive Neuroscience, 9, 555-604.

Nunn, J. A., Postma, P., \& Pearson, R. (2001). Developmental prosopagnosia: Should it be taken at face value? Neurocase, 7, 15-27.

Perrett, D. I., Rolls, E. T., \& Caan, W. (1982). Visual neurones responsive to faces in the monkey temporal cortex. Experimental Brain Research, 47, 329-342.

Puce, A., Allison, T., \& McCarthy, G. (1999).

Electrophysiological studies of human face perception: III. Effects of top-down processing on face-specific potentials. Cerebral Cortex, 9, 445-458.

Riesenhuber, M., Jarudi, I., Gilad, S., \& Sinha, P. (2004). Face processing in humans is compatible with a simple shape-based model of vision. Proceedings of the Royal Society of London, B, Biological Sciences, 271, S448-S450.

Sergent, J., \& Signoret, J.-L. (1992). Varieties of functional deficits in prosopagnosia. Cerebral Cortex, 2, 375-388.

Tanaka, J. W., \& Farah, M. J. (1993). Parts and wholes in face recognition. Quarterly Journal of Experimental Psychology, A, Human Experimental Psychology, 46A, 225-245.

Tanaka, J. W., \& Farah, M. (2003). The holistic representation of faces. In M. A. Peterson \& G. Rhodes (Eds.), Perception of faces, objects and scenes: Analytic and holistic processes. New York: Oxford University Press.

Tanaka, J. W., \& Sengco, J. A. (1997). Features and their configuration in face recognition. Memory \& Cognition, 25, 583-592.

Yin, R. (1969). Looking at upside down faces. Journal of Experimental Psychology, 81, 141-145.

Young, A. W., Hellawell, D., \& Hay, D. C. (1987). Configurational information in face perception. Perception, 16, 747-759.

Yovel, G., \& Kanwisher, N. (2004a). Face perception: Domain specific, not process specific. Neuron, 44, 1-20.

Yovel, G., \& Kanwisher, N. (2004b). Face perception engages a domain-specific system for processing both configural and part-based information about faces [Abstract]. Journal of Vision, 4, 133a. 